\title{
Calculating Frequency-Dependent Inductance of VLSI Interconnect by Complete Multiple Reciprocity Boundary Element Method*
}

\author{
Changhao Yan \\ Wenjian $\mathrm{Yu}$ \\ Dept. of Comp. Sci. \& Tech. Dept. of Comp. Sci. \& Tech. \\ Tsinghua University \\ Beijing, 100084 \\ Tel: 8610-6279-5428 \\ Fax: 8610-6278-1489 \\ yanch02@mails.tsinghua.edu.cn
}

\author{
Zeyi Wang \\ Dept. of Comp. Sci. \& Tech. \\ Tsinghua University \\ Beijing, 100084 \\ Tel: $8610-6279-5428$ \\ Fax: 8610-6278-1489 \\ wangzy@mail.tsinghua.edu.cn
}

\begin{abstract}
A complete multiple reciprocity method (CMRM), usually for the eigenvalue analysis of Helmholtz equation, is introduced to the BEM for frequency-dependent inductance extraction. Several approaches are proposed to resolve the problem of "ill-conditioned" series encountered when applying the CMRM practically. Using the BEM combined with CMRM, the major operations of calculating the numerical integrals for a frequency point become reusable, so that inductance extraction for a frequency range is greatly accelerated. Numerical results verify the accuracy and efficiency of the proposed method.
\end{abstract}

\section{INTRODUCTION}

The parasitic inductance of VLSI interconnect has become very important as the operating frequency of circuit keeps increasing [1]. Since the inductance is frequencydependent, inductance extraction for a range of frequency points is usually needed for accurate simulation and verification. Besides, with the inductances and resistances for a frequency range, the quality factor of an inductor component in RF circuit can be calculated [2]. Therefore, inductance extraction for a frequency range, not only for a single frequency point, becomes another important research topic.

There are two categories of methods for calculating frequency-dependent inductance. The first category employs the interpolation method $[3,4]$. For example, paper [4] uses a coupled circuit method to calculate the lowfrequency inductance and the inversion of capacitance matrix for high-frequency inductance, and then forms the full-frequency inductance curve with an interpolation formula. Although this category of methods has high computational speed, their reliability and accuracy is very limited because only several interpolation points for very low frequency and very high frequency are computed accurately.

${ }^{*}$ This work is supported by the China National Science Foundations under Grant 90407004 and 60401010 . And, it is partly supported by National High Technology Research and Development Program of China (No. 2004AA1Z1050).
The other category is straightforward. To obtain the inductance for a range of frequency, a method to extract the inductance must run repeatedly for all sampling points throughout the frequency range. The methods suitable for inductance extraction at arbitrary frequency include FastHenry, a volume integral method [5], FastImp, a boundary element method (BEM) [6], etc. These methods have high accuracy for inductance extraction at any frequency point, but computational speed is relatively slow. The BEM for inductance extraction proposed recently employs much fewer unknowns and is suitable for a wideband simulation [6]. This method has been implemented as a software prototype named FastImp, available via the Internet [8]. Compared with FastHenry, the FastImp is faster. However, using the FastImp, the time of inductance extraction for a frequency range is still very long, which approximates to the number of frequency points times the computational time for one frequency point. Speeding up the inductance extraction based on $\mathrm{BEM}$ for a range of frequency is the main target of this paper.

In the inductance extraction based on BEM, the time is mainly spent on calculating all kinds of numerical integrations and solving the linear equation system $A x=b$. The CPU time of solving $A x=b$ can be reduced greatly, if an efficient iterative method, such as GMRES, is applied. So, the time of numerical integrations occupies the major part of the whole. For example, computing the example wire.inp in one frequency point, FastImp needs $4.62 s$ in total, where solving $A x=b$ costs only $0.58 \mathrm{~s}$ and the frequency-dependent numerical integration (with pFFT acceleration) costs 3.95s [8]. These numerical integrals can be divided into two types. One type is frequency-independent, where the integral kernel is $1 / r$ or its derivative, coming from the fundamental solution of the Laplace equation in dielectrics. Their results can be reused among all sampling frequencies. The other type is frequency-dependent, where the integral kernel is $e^{-j k r} / r$ or its derivative, coming from the fundamental solution of the Helmholtz equation in conductors, where $k$ is a complex number and means the wave number in physics. The latter type must be recomputed in every 
frequency point. Furthermore, the frequency-dependent integrations are much more expensive than the frequencyindependent integrations in CPU time, since computing the $e^{-j k r}$ is very arduous. For example, on a PC with an Intel Pentium4 1.8G CPU, the time ratio of integrating $e^{-j k r} / r$ to $1 / r$ is about $7: 1$. So how to shorten the computing time of the second type of integration becomes a key problem for inductance extraction based on BEM, especially for many frequency points.

On the other hand, a multiple reciprocity method (MRM) was proposed in [7] for boundary value problems of the Helmholtz equation. Then, it is applied to the eigenvalue analysis of Helmholtz equation successfully [9], which reduces the computational expense greatly. In MRM, the integral $\int e^{-j k r} / r$ can be transformed into a series $\sum_{j=0}^{N} g_{j}(k) \int f_{j}(r)$, where $\int f_{j}(r)$ is a frequencyindependent series and $g_{j}(k)$ is a frequency-dependent series. Based on this transformation, one can compute and save the numerical integrals of $\int f_{j}(r)$ at first, and then for a different sampling frequency, i.e., a different $\mathrm{k}, \int f_{j}(r)$ need not to be computed again. Instead, only the coefficients of $g_{j}(k)$ are calculated for each frequency point, and the integral $\int f_{j}(r)$ need to be computed only once. Therefore, the computational time of the frequencydependent integral $\int e^{-j k r} / r$ can be greatly reduced for the other frequency points except the first one.

In this paper, the MRM is applied to the interior boundary value problem (BVP) of Helmholtz equation in 3-D inductance extraction. To avoid the spurious eigenvalue problem caused by the MRM formulation [10], a complete MRM (CMRM) formulation proposed by [11] is actually adopted in our method. Although it was proved that the series in MRM or CMRM are convergent, a problem of "ill-conditioned" series is still encountered when we apply the MRM formulation to 3-D inductance extraction. Because the $k$ (frequency) and $r$ (distance) are both near to 1 in the eigenvalue analysis with $\operatorname{MRM}[10,12,13]$, this problem did not emerge in literatures. But in the inductance extraction of VLSI interconnect, the $r$ usually varies from $1 \mathrm{~mm}$ to $90 \mathrm{~nm}$ and the frequency $f$ varies from $1 \mathrm{~Hz}$ to $10 \mathrm{GHz}$. Therefore, several approaches are proposed in this paper to handle the problem of "ill-conditioned" series. Several interconnect structures are calculated with the BEM combined with CMRM. Numerical results show that our method has high accuracy as that of FastImp, and the CMRM exhibits large speedup for inductance extraction with many frequency points.

\section{Multiple Reciprocity Boundary Element METHOD}

In this section, we will briefly introduce the MRM [9] and CMRM for the BEM used for inductance extraction.

\section{A. Conventional MRM Formulation}

In a 3 -D closed domain $\Omega$ surrounded by the boundary $\Gamma$, potential $u$ satisfies the Helmholtz equation:

$$
\nabla^{2} u+k^{2} u=0
$$

where $k$ is the wave number. In the inductance extraction problem with MQS (magnetoquasistatics) assumption, $k=\sqrt{-j \omega \mu \sigma}$, where $\mathrm{j}, \omega, \mu$ and $\sigma$ are the imaginary unit, angular frequency, permeability and conductivity respectively. $\mu$ and $\sigma$ are constant.

From (1), it is easy to obtain the boundary integral equation (BIE) as follows:

$$
c u+\int_{\Gamma}\left(u q_{1}^{*}-q u_{1}^{*}\right) d \Gamma=0
$$

where $\quad u_{1}^{*}=\frac{e^{-j k r}}{4 \pi r}, \quad q_{1}^{*}=\frac{\partial u_{1}^{*}}{\partial n}, \quad \mathrm{c}$ is constant depending on the position of the source point and where the equation is considered, $r$ is the distance between the source and filed point and $\vec{n}$ is the outward normal on the boundary. The Eq. (2) is called complex-value formulation for the Helmholtz equation in this paper. The FastImp belongs to a direct BEM because it utilizes this formula [15].

Moving the second term on the left hand side (l.h.s.) of (1) to the right, one gets a Poisson equation as:

$$
\nabla^{2} u=-k^{2} u \text {. }
$$

Similarly, a BIE can be obtained:

$$
c u+\int_{\Gamma}\left(u q_{0}^{*}-q u_{0}^{*}\right) d \Gamma=k^{2} \int_{\Omega} u u_{0}^{*} d \Omega,
$$

where $u_{0}^{*}=\frac{1}{4 \pi r}, \quad q_{0}^{*}=\frac{\partial u_{0}^{*}}{\partial n}$.

Although in (4), the Laplace fundamental solution $u_{0}^{*}, q_{0}^{*}$ is frequency-independent, for the volume integral term on the r.h.s. of (4), directly applying it leads to volume discretization needed, while the absence of the volume discretization is thought as the key advantage of the BEM compared with other volume integral methods.

The MRM is known as a powerful conversion scheme from the domain integral to the boundary [7, 9]. Adopting the higher order fundamental solution of the Laplace equation:

$$
\nabla^{2} u_{j+1}^{*}=u_{j}^{*}=\frac{1}{4 \pi r} \frac{r^{2 j}}{(2 j) !}, \quad q_{j}^{*}=\frac{\partial u_{j}^{*}}{\partial n}, \quad j=0,1,2, \ldots,
$$

the domain integral of (4) can be transformed into:

$$
\begin{aligned}
& \int_{\Omega} u \nabla^{2} u_{1}^{*} d \Omega=\int_{\Gamma}\left(u q_{1}^{*}-q u_{1}^{*}\right) d \Gamma+\int_{\Omega} u_{1}^{*} \Delta u d \Omega \\
& =\int_{\Gamma}\left(u q_{1}^{*}-q u_{1}^{*}\right) d \Gamma-k^{2} \int_{\Omega} u_{1}^{*} u d \Omega,
\end{aligned}
$$

where the first transformation uses the second Green theorem and the second transformation uses (3). Substituting (5) into the r.h.s. of (4), and arranging it, one obtains:

$$
\begin{aligned}
& c u+\int_{\Gamma}\left(u q_{0}^{*}-q u_{0}^{*}\right) d \Gamma+ \\
& -k^{2} \int_{\Gamma}\left(u q_{1}^{*}-q u_{1}^{*}\right) d \Gamma=\left(-k^{2}\right)^{2} \int_{\Omega} u_{1}^{*} u d \Omega .
\end{aligned}
$$

Repeating similar computation leads to

$$
\begin{aligned}
& c u+\sum_{j=0}^{N}\left(-k^{2}\right)^{j}\left[\int_{\Gamma}\left(u q_{j}^{*}-q u_{j}^{*}\right) d \Gamma\right] \\
& =(-1)^{N}\left(k^{2}\right)^{N+1} \int_{\Omega} u_{N}^{*} u d \Omega \approx 0 .
\end{aligned}
$$

For sufficient large $\mathrm{N}$, if $r$ and $k$ are bounded, the domain integral on the r.h.s. of (6) becomes negligible [7]. Eq. (6) is the basic formula in the MRM.

Note that (6) does not satisfy the Sommerfeld radiation condition at infinity and cannot be employed for an unbounded domain such as: exterior BVP, But this is not a problem in inductance extraction for that all the domains in consideration are within bounded conductors.

\section{B. Complete MRM Formulation}

The MRM formulation mentioned above is called conventional MRM formulation, where both $u_{j}^{*}, q_{j}^{*}$ are real. If 
the conventional MRM formulation is used for eigenvalue problem, spurious eigenvalue will occur [10]. Ref. [11] proposed a complete MRM formulation as follows:

$$
\begin{aligned}
& u_{j}^{*}=\frac{1}{4 \pi}\left[\frac{1}{r} \frac{r^{2 j}}{(2 j) !}-i k \frac{r^{2 j}}{(2 j+1) !}\right], \quad q_{j}^{*}=\frac{\partial u_{j}^{*}}{\partial n}, \quad j=0,1,2, \ldots, \\
& u^{*}=\sum_{j=0}^{N}\left(-k^{2}\right)^{j} \int_{\Gamma} u_{j}^{*} d \Gamma, \quad q^{*}=\sum_{j=0}^{N}\left(-k^{2}\right)^{j} \int_{\Gamma} q_{j}^{*} d \Gamma,
\end{aligned}
$$

by which this spurious eigenvalue problem can be solved perfectly. Moreover, they pointed out that the final series form of the kernel in (complete) MRM simply converges to corresponding kernels in the complex-value formulation [11].

To avoid the possible spurious eigenvalue problem caused by the MRM formulation, the CMRM formulation is adopted in our method for inductance extraction. When applying the CMRM to the direct BEM in [15], we only need to replace the $u_{1}^{*}$ and $q_{1}^{*}$ in (2) with the $u^{*}$ and $q^{*}$ in (7), respectively. It should also be pointed out that the CMRM formulation actually expends more computational resources than the conventional MRM, but its good numerical stability makes us choose it.

\section{Difficulties And Solutions of Inductance EXTRACTION USING CMRM}

The numerical difficulties produced by directly applying CMRM formulation will be discussed in the first subsection. Then a set of methods to overcome these difficulties are proposed in next three subsections. In the last subsection a recursion formulation for accelerating computation is proposed.

\section{A. Ill-conditioned Series}

In numerical methods textbooks, the series like:

$$
e^{-x}=\sum_{j=0}^{n} \frac{(-x)^{n}}{n !}
$$

is called ill-conditioned series [14]. In such a kind of series, large terms which are many orders of magnitude larger than the sum of the series cancel out with each other. So very small errors in these large terms produce large errors in the final result.

Reexamining the CMRM formulation, one will find the series $u_{j}^{*}, q_{j}^{*}$ and $\left(-k^{2}\right)^{j}$ are all ill-conditioned. For a slightly large $k$ or $r$, numerical computations are impossible. Eigenvalue analysis doesn't encounter this difficulty because it guarantees both the $k$ and $r$ are near to 1 [10]. But in VLSI circuits, this cannot be satisfied. The numerical difficulties are in three aspects. First, The very small absolute value of $r$. In VLSI circuits, the unit of distances is micron or nanometer. For avoiding the fussy unit conversion, the International System of Units is usually adopted. Then $r$ will be a very small number. Substituting it into ill-conditioned series directly will cause the underflow. Second, The wide variation range of the $r$. In VLSI interconnects, the ratio of the dimension in the length to the one in the section is up to several decades. This problem cannot be solved by simple unit conversion.
Finally, The wide variation range of the $k$. The frequencies from $1 h z$ to $10 G h z$ are taken into consideration. If conductors are coppers, the $k$ varies from 0 to $10^{6}$.

If $x$ is a large number, computing the series (8) directly is not feasible, but its analysis result is known and approaches zero. In physics, the series is called exponential decay. So it is possible to compute it by other approximate method or even to ignore it. If $x$ nears to 1 , however, the series converges quickly. This feature is valuable.

\section{B. Normalization of the Distance $r$}

Without loss of generality, let's consider the real part of the $u^{*}$. Introducing a average distance $r_{a v g}$, one obtains:

$$
\begin{aligned}
u_{\text {real }}^{*} & =\frac{1}{4 \pi} \sum_{j=0}^{N}\left(-k^{2}\right)^{j} \int \frac{1}{r} \frac{r^{2 j}}{(2 j) !} d \Gamma \\
& =\frac{1}{4 \pi} \sum_{j=0}^{N}(-1)^{j}\left(k r_{\text {avg }}\right)^{2 j} \int \frac{1}{r} \frac{r_{r e l}^{2 j}}{(2 j) !} d \Gamma,
\end{aligned}
$$

where: $r_{r e l}=\frac{r}{r_{a v g}}$.

From the discussion above, the series $\sum_{j=0}^{N} \int \frac{1}{r} \frac{r_{r e l}^{2 j}}{(2 j) !} d \Gamma$ can converge very quickly if choosing an appropriate $r_{a v g}$ to keeping $r_{r e l}$ near to 1 . This transformation has two effects. The one is solving the first problem, i.e., the very small absolute distance. The other is partially solving the second and third problem, combining the variation of the $k$ or $r$ respectively into the variation of the $k r_{\text {avg }}$ together.

Note that a different $r_{a v g}$ is needed between any two panels. That means $r_{a v g}$ is a matrix indeed, but it is frequency-independent. In the program of this paper, the formula of computing $r_{a v g}$ is the average distance among the distances between any corner of the integral panel and the source point.

\section{Localization of the Near Field}

If $k r_{\text {avg }}$ is a large number, a window with size $W$ is given. When $k r_{\text {avg }}<W, u_{\text {real }}^{*}$ can be computed by series formula (9) directly. Then two questions should be answered, that is how large of the window is big enough and how many terms of the series are needed for a given windows size $W$.

Let us consider the meaning of $k r_{a v g}$ in physics. Under MQS assumption, one obtains $k=(1-j) k_{\text {real }}$. For simplicity, only considering $k_{\text {real }} r_{\text {avg }}$, based on the fact that:

$$
k_{\text {real }}=\sqrt{\frac{2}{\omega \mu \sigma}}=\frac{1}{\delta},
$$

where $\delta$ is the skin depth in the angle frequency $\omega$, we get:

$$
k_{\text {real }} r_{a v g}=\frac{r_{a v g}}{\delta} .
$$

In physics, the Eq. (10) indicates that the $k_{\text {real }} r_{\text {avg }}$ is the ratio of the average distance between the panel and the source point to the skin depth in current frequency. It is nondimensional. In the electromagnetic wave theory, the $k_{\text {real }}$ is termed attenuation constant. If the filed point is five times skin depth away from the source, the amplitude attenuation is under one percent [2](pp.253). So for the engineering application, letting $W=5 \sim 6$ is enough. 
Next, considering that the real part of $u^{*}$ simply converges to $\cos (k r)$, from the convergence curve of $\cos \theta$, one obtains the relationship between the length $N$ of the series and the windows size $W$ in Table I.

From the Table I, if $W=2 \pi$ (a wave length), only letting $N=11$, four correct decimals can be obtained. If $W$ is smaller, for the same accuracy, the $N$ is smaller too.

\section{Approximate Calculation of the Far Field}

If $k r_{\text {avg }}>W$, from the discussion above, the effect of the source is feeble, so it is possible to sum this effect in a coarse way.

Considering the fact that the complete MRM formulation simple converges to the kernel in the complex-value formulation, one can directly compute $u^{*}$ by the integral of the kernel of the Helmholtz equation as follow:

$$
u^{*}=\frac{1}{4 \pi} \int_{\Gamma} \frac{e^{-j k r}}{r} d \Gamma \approx \frac{e^{-j k r_{a v g}}}{4 \pi} \int_{\Gamma} \frac{1}{r} d \Gamma=e^{-j k r_{a v g}} \cdot u_{0} .
$$

The reason of the $\approx$ in $(11)$ is that now that the source point is far away from the integral panel, it is meaningless to exactly sum the integration on every $r$ which is the distance between a guass point in the integral panel and the source point, so a average distance between the integral panel and the source point is enough.

Notice that this approximate formulation also includes the arduous $e^{-j k r_{a v g}}$, but it is computed only on a distance $r_{a v g}$, not like the direct integral formula where computation of $e^{-j k r}$ is needed on every gauss point. More convenient, the $u_{0}$ is right the first term of the series $u_{j}^{*}$, so the additional computation is unnecessary.

\section{E. Fast Computation of the Series}

Direct computing the power series as the formulation proposed above will waste lots of CPU time. Fortunately, it is easy to transform the direct form of the series into recursion formula from which amounts of time can be saved. Taking the real part of $u_{j}^{*}$ as example, one obtain the recursion form as follow:

$$
u_{j+1, \text { real }}^{*}=\frac{1}{r} \frac{r_{r e l}^{2 j}}{(2 j) !}=\frac{r_{r e l}^{2}}{(2 j+2)(2 j+1)} u_{j, r e a l}^{*},
$$

where $u_{0, \text { real }}^{*}=\frac{1}{r}$.

Moreover, in order to accelerate the computation, the coefficient $\frac{1}{(2 j+2)(2 j+1)}$ can be computed and saved in a table before. By now, on a gauss point, one term of the series added needs only two extra multiply operators.

Note that when the source point locates in the integral panel, $u_{0, \text { real }}^{*}$ is weak singular integral and $q_{0}^{*}$ is strong singular integral. Both of them need special treatments.

\section{Numerical Results}

The proposed method based on CMRM is implemented in $\mathrm{C}++$ language, and the original BEM for inductance extraction is as same as that proposed in [15]. In the following experiments, our method is compared with the original direct BEM. The former is denoted by CMRM and the latter by ODBEM. In the FastImp, which is also based on the direct BEM of [15], a precorrected-FFT (pFFT) acceleration algorithm is employed [6]. However, pFFT also handles the boundary integrals, and conflicts with the CMRM. Therefore, in the ODBEM, only a direct solver of linear equation system is implemented, which does not affect the performance evaluation of the CMRM for extraction with many frequency points. Our CMRM and ODBEM are used to extraction frequency-dependent inductance for three interconnect structures. In the computations, conductor surface is discretized into constant rectangular elements, with collocation point located in the center. To guarantee the accuracy of near singular integrals, a 2D Guass-Legendre integral scheme with $20 \times 20$ integral points is used.

\section{A. A Single Rectangle Wire}

A straight conductor wire is $8 \mu \mathrm{m}$ long, $1 \mu \mathrm{m}$ wide and $1 \mu \mathrm{m}$ thick. The surface of this wire is discretized into $160(4 \times 4 \times 8)$ panels, as shown in Fig. 1 . The number of unknowns is 1122 .

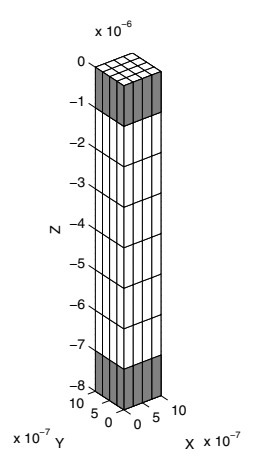

Fig. 1. A Single Rectangle Wire

The red panels surrounding the contacts are for summing the current in the conductor with the high frequency method and the $F_{h}=15 G h z$ [15]. Calculating the inductance from $1.5 \mathrm{Mhz}$ to $40 \mathrm{Ghz}$ with the ODBEM, one obtains the inductance curve in Fig. 2. Fig. 3 is the result from [15] (pp.110), where the frequency range is from $1 \mathrm{~Hz}$ to $10^{20} \mathrm{hz}$. Note that the highest frequency compared is only up to $40 \mathrm{Ghz}$.

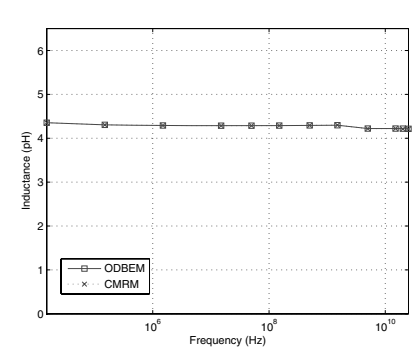

Fig. 2. Inductance

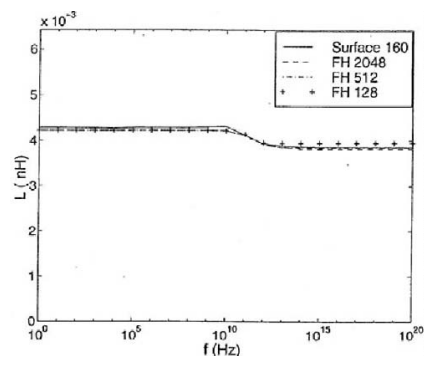

Fig. 3. Inductance(from [15])
In CMRM, the window size $W=2 \pi$ and the length of the series $N=12$. From Fig. 2 both results from the CMRM and the ODBEM coincide with each other very well on the whole frequency range, and their results coincide with the data from [15], i.e. Fig. 3. 
TABLE I

Relation Between $W$ AND $N$

\begin{tabular}{|c|c|c|c|c|c|c|c|c|c|c|c|c|c|}
\hline $\mathrm{N}$ & 1 & 2 & 3 & 4 & 5 & 6 & 7 & 8 & 9 & 10 & 11 & 12 & exact \\
\hline $\mathrm{W}=\pi / 2$ & 0000 & -0.23 & 0200 & .000 & 000 & & & & & & & & 000 \\
\hline $\mathrm{W}=\pi$ & 0000 & -3.9348 & 0.1239 & 4 & 760 & 18 & 99 & & & & & & תמת \\
\hline$W=3 \pi / 2$ & .0000 & -10.1033 & 0.4439 & 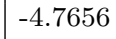 & 7 & 4 & 0.0 & 26 & 02 & & & & -0.0000 \\
\hline $\mathrm{W}=2 \pi$ & 1.0000 & -18.7392 & 46.2002 & -39.2566 & 20.9880 & -5.4382 & 2.4653 & 0.7509 & 1.0329 & 0.9965 & 1.0003 & 0.99998 & 1.0000 \\
\hline
\end{tabular}

Note that when $f=15 G h z$, the skin depth $\delta=0.54 \mu m$ and $r_{\text {avg }}<3.3 \mu \mathrm{m}$. That means the approximate formulation has been employed when the distance between two panels is larger than $3.3 \mu \mathrm{m}$. At this frequency, the results from the ODBEM and the CMRM still have the accuracy of three correct decimals.

In order to compare the speed between the ODBEM and the CMRM clearly, we briefly summarize the whole steps in the program listed as follows.

1. calculate the integral of the Laplace kernel related

2. calculate the integral of the Helmholtz kernel related (repeated in ODBEM, partly repeated in CMRM)

3. generate the linear system (repeated)

4. solve the linear system ( repeated)

5. postporcess. Obtain impedance matrix ( repeated)

The main differences lie in step 2. The ODBEM directly compute numerical integrals. The CMRM divides this step into three steps:

1. compute $r_{\text {avg }}$ matrix

2. generate and save the series

3. sum the series for a given $k$ (repeated)

The time result is listed in Table II. The unit is second. This program is run on a desktop PC with a Pentium4 1.8G CPU and 256M RAM. The symbol asterisk indicates that the step should be recomputed at different frequency points.

TABLE II

CPU Time of Single Wire Example

\begin{tabular}{|c|c|c|c|c|c|c|c|c|c|}
\hline & $\begin{array}{l}\text { Lap. } \\
\text { Int. }\end{array}$ & & Helm & Int & & $\begin{array}{l}\text { Gen. } \\
A x=b\end{array}$ & & & $\begin{array}{l}\text { Post } \\
\text { proc. }\end{array}$ \\
\hline ODBEM & \multirow[b]{2}{*}{1.766} & & 11.14 & & \multirow{2}{*}{\multicolumn{2}{|c|}{$0.125^{*}$}} & & \multirow[b]{2}{*}{$0.000^{*}$} \\
\hline MR & & \begin{tabular}{|l|}
$R_{a v g}$ \\
0.000 \\
\end{tabular} & \begin{tabular}{|l|} 
Serial \\
9.234
\end{tabular} & \begin{tabular}{|c|c|} 
& Combin \\
\end{tabular} & & & & & \\
\hline
\end{tabular}

From the Table II, we can conclude:

1. The time ratio of the Helmholtz integral to the Laplace integral is about 7:1(11.141:1.766). For only one conductor in the example, this ratio is also the CPU time ratio of computing the kernel $e^{-j k r} / r$ to $1 / r$. Obviously, the Helmholtz kernel is much more expensive than the Laplace kernel on the CPU time.

2. From the second frequency point, the time of the integral is near to zero $(0.156 \mathrm{~s})$ by the ODBEM, and the rest is only the time of solving $A x=b$.

3. Even for the first frequency point, CMRM (9.390s) is still a little faster than ODBEM (11.141s).

The same conclusion can be obtained from the Fig. 4, where the horizontal coordinate axis stands for the number of the sampling frequency points and the vertical coordinate axis stands for the total time. The inductances on twelve sampling frequency points are computed. The advantage of saving time by CMRM is very apparent.

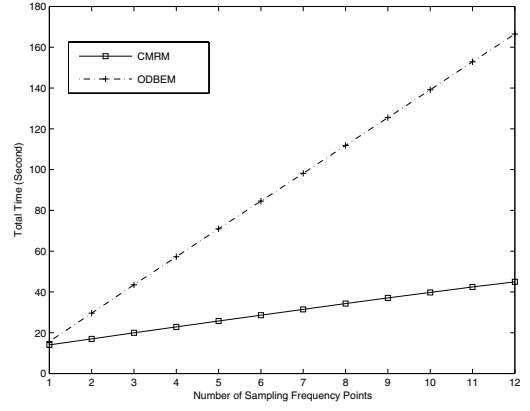

Fig. 4. CPU Time Comparison

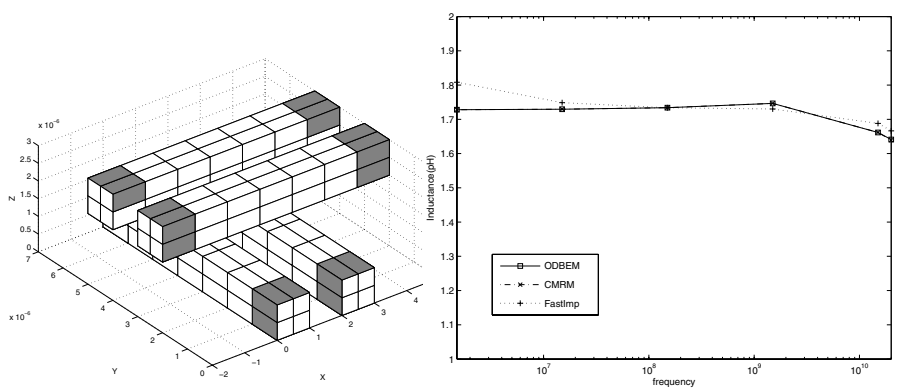

Fig. 5. A $2 \times 2$ Bus

Fig. 6. Mutual Inductance of a Parallel Wire in 2x2 Bus

\section{B. A $2 \times 2$ Bus}

The dimension and discretization of the bus2x2 example is shown in Fig. 5.

The numbers of panels and unknowns are 256 and 1800 respectively. Because there are four conductors, the result of the inductance should be a $4 \times 4$ matrix.

Table III gives this results at $f=15 G h z$. The unit is $p H$. For comparison, the result of the Fastimp is introduced. Fig. 6 shows the mutual inductance of parallel conductors i.e. the location $(1,2)$ in inductance matrix from $1.5 \mathrm{Mhz}$ to $40 \mathrm{Ghz}$. All of them coincide with each other very well. The result of CPU time Comparison is not listed here because it is similar to the first example.

\section{A $1 \times 1$ Bus}

This example describes the relation between accuracy and window size $W$. The dimension and discretization of the bus $1 \mathrm{x} 1$ example is shown in Fig. 7

The number of panels and unknowns are 320 and 2240 respectively. At $f=15 G h z$, applying different window size $W$ and length of series $N$, the result of the selfinductance in a conductor are listed in Table IV.

Take the result from the ODBEM as the exact result. From the Table IV, one concludes that at high frequency, even if $N=5$, the resistance has a relative error about $10 \%$ and the inductance has a relative error about $3 \%$. It 
TABLE III

InduCtance Matrix of a 2X2 Bus When $f=15 G h z$

\begin{tabular}{|c|c|c|c|c|c|c|c|c|c|c|}
\hline \multicolumn{3}{|c|}{ CMRM } & \multicolumn{4}{|c|}{ ODBEM } & \multicolumn{4}{|c|}{ FastImp } \\
\hline 3.419 & 1.66187 & $7.16 \mathrm{e}-4 \quad 7.32 \mathrm{e}-4$ & 3.4198 & 1.66161 & $7.16 \mathrm{e}-4$ & $7.32 \mathrm{e}-4$ & 3.4697 & 1.68804 & $7.81 \mathrm{e}-4$ & $7.10 \mathrm{e}-4$ \\
\hline 1.66 & 3.41942 & $7.16 \mathrm{e}-4 \quad 7.32 \mathrm{e}-4$ & 1.661 & 3.41989 & $7.16 \mathrm{e}-4$ & $7.32 \mathrm{e}-4$ & 1.68802 & 3.4697 & $7.73 \mathrm{e}-4$ & $7.08 \mathrm{e}-4$ \\
\hline $7.32 \mathrm{e}-4$ & $7.16 \mathrm{e}-4$ & $3.41942 \quad 1.66187$ & $7.32 \mathrm{e}-4$ & $7.16 \mathrm{e}-4$ & 3.41989 & 1.66161 & $8.02 \mathrm{e}-4$ & $6.59 \mathrm{e}-4$ & 3.47036 & 1.68878 \\
\hline $7.32 \mathrm{e}-4$ & $7.16 \mathrm{e}-4$ & 1.661873 .41942 & 7.32e-4 & $7.16 \mathrm{e}-4$ & 1.66161 & 3.41989 & $8.24 \mathrm{e}-4$ & $6.76 \mathrm{e}-4$ & 1.68877 & 3.47036 \\
\hline
\end{tabular}

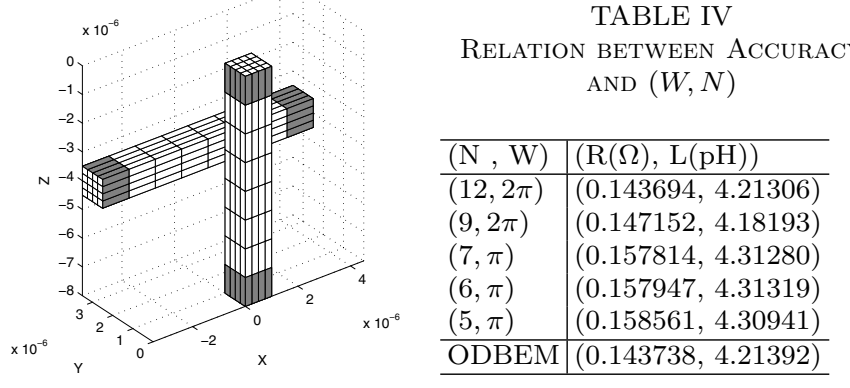

Fig. 7. A $1 \mathrm{x} 1$ Bus

is meaningful for saving double size of spaces with acceptable loss of the accuracy.

\section{Conclusions}

In the inductance extraction with BEM, much of the CPU time is spent on numerical integrals. A CMRM, usually for eigenvalue analysis of Helmholtz equation, is introduced to the BEM based inductance extraction. To solve the problem of ill-conditioned series caused by large variation of parameter $k$ and $r$ in inductance extraction, several approaches are proposed for CMRM, including the normalization of the distance $r$, the localization of the near field and the approximate computation for the far field. With the CMRM and proposed efficient techniques, the computation of frequency-dependent integrals in BEM becomes reusable. Numerical experiments show that the proposed method greatly speeds up the inductance extraction for a frequency range, while preserving high accuracy.

\section{ACKNOWLEDGEMENTS}

The authors would like to thank Prof. J. T. Chen, Dr. W. Yeih of Taiwan Ocean University for useful discussion.

\section{REFERENCES}

[1] A. B. Kahng, S. Muddu, "Analytical delay model for RLC interconnects," IEEE Trans. on CAD, vol. 16, pp. 1507-1514, 1997.

[2] Bhag Singh Guru, Huseyin R. Hiziroglu, Electromagnetic Field Theory Fundamentals, pp. 365-367. PWS Publishing Company, 1998.

[3] M. J. Tsuk, J. A. Kong, "A hybid method for the calculation of the resistance and inductance of transmission lines with arbitrary cross sections," IEEE Trans. on MTT., vol. 39, pp. 1338-1347, 1991.

[4] S. Fang, X. Tang, Z. Wang and X. Hong, "A simplified hybrid method for calculating the frequency-dependent inductances of transimission lines with rectangular cross section," Proc. of $A S P-D A C$, pp. 453-456, 2000, Yokohama, Japan.

[5] M. Kamon, M. J. Tsuk, J. White, "Fasthenry: a multipoleaccelerated 3-d inductance extraction program," IEEE Trans, on $M T T$, vol. 42, no. 9, pp. 1750-1758, 1994.
[6] Z. Zhu, B. Song, J. White, "Algorithms in fastimp: a fast and wideband impedance extraction program for complicated 3-d geometries," DAC, pp. 712-717, 2003.

[7] A. J. Nowak and C. A. Brebbia, "Solving helmholtz equation by boundary elements using the multiple reciprocity method," Computers and Experiments in Fluid Flow, pp. 265-270, 1989.

[8] Z. Zhu, B. Song, J. White, FastImp user's guid, http://relweb.mit.edu/vlsi/codes.htm.

[9] N. Kamiya and E. Andoh, "Helmholtz eigenvalue analysis by boundary element mehtod," Journal of Sound and Vibration, vol. 160, no. 2, pp. 279-287, 1993.

[10] N. Kamiya, E. Andoh and K. Nogae, "Three-dimensional eigenvalue analysis of the helmholtz equation by multiple reciprocity boundary element method," Advances in Engineering Software, vol. 16, pp. 203-207, 1993.

[11] W. Yeih, J. T. Chen, K. H. Chen and F. C. Wong, "A study on the multiple reciprocity method and complex-valued formulation for the helmholtz equation," Advances in Engineering Software, vol. 29, no. 1, pp. 1-6, 1998.

[12] N. Kamiya, E. Andoh, K. Nogae, "A new complex-valued formulation and eigenvalue analysis of the helmholtz equation by boundary element method," Advances in Engineering Software, vol. 26, pp. 219-227, 1996.

[13] J.T. Chen and F.C. Wong, "Analytical derivations for onedimensional eigenproblems using dual boundary element method and multiple reciprocity method," Engineering Analysis with Boundary Elements, vol. 20, pp. 25-33, 1997.

[14] Germund Dahlquist, Ake Bjorck, Translated by Ned Anderson, Numerical Methods, pp. 75-78. Englewood Cliffs, N.J.: Prentice-Hall, Inc., 1974.

[15] J. F. Wang, A new surface integral formulation of EMQS impedance extraction for 3-D structures. PhD thesis, MIT, 1999. 